\title{
Prognostic value of aspartate transaminase to alanine transaminase (De Ritis) ratio in solid tumors: a pooled analysis of 9,400 patients
}

This article was published in the following Dove Press journal: OncoTargets and Therapy

\author{
Jiayuan $\mathrm{Wu}^{\mathrm{l}, *}$ \\ Lin Chen ${ }^{2, *}$ \\ Yufeng Wang ${ }^{3, *}$ \\ Wenkai $\operatorname{Tan}^{4}$ \\ Zhe Huang $^{5}$ \\ 'Clinical Research Center, The Affiliated \\ Hospital of Guangdong Medical \\ University, Zhanjiang 52400I, Guangdong \\ Province, People's Republic of China; \\ ${ }^{2}$ Department of Cardiac Surgery, The \\ Affiliated Hospital of Guangdong Medical \\ University, Zhanjiang 52400I, Guangdong \\ Province, People's Republic of China; \\ ${ }^{3}$ School of Public Health, Guangdong \\ Medical University, Zhanjiang 524023, \\ Guangdong Province, People's Republic \\ of China; ${ }^{4}$ Department of \\ Gastroenterology, The Affiliated Hospital \\ of Guangdong Medical University, \\ Zhanjiang 52400I, Guangdong Province, \\ People's Republic of China; ${ }^{5}$ Department \\ of Gastrointestinal Surgery, The Affiliated \\ Hospital of Guangdong Medical \\ University, Zhanjiang 52400I, Guangdong \\ Province, People's Republic of China
}

*These authors contributed equally to this work

Correspondence: Jiayuan Wu

Department of Clinical Research, The Affiliated Hospital of Guangdong Medical University, No 57, South of People's Avenue, Zhanjiang 52400I, Guangdong,

People's Republic of China

Tel +8607592387506

Fax +8607592387612

Email 87537665@qq.com

Zhe Huang

Department of Gastrointestinal Surgery,

The Affiliated Hospital of Guangdong

Medical University, No 57, South of

People's Avenue, Zhanjiang 52400I,

Guangdong, People's Republic of China

Email3960186I8@qq.com
Background: Numerous studies have reported the association between pretreatment serum aspartate transaminase to alanine transaminase (AST/ALT) ratio and prognosis in multiple cancers. However, the results remain controversial and no consensus has been reached. Thus, we conducted this meta-analysis to quantitatively assess the prognostic value of pretreatment AST/ALT ratio in solid tumors.

Methods: A systematic literature search was conducted by using PubMed, EMBASE, Web of Science, Cochrane Library, and Wanfang databases, as well as several trial registry platforms, including ClinicalTrials.gov, WHO International Clinical Trials Registry Platform, and Chinese Clinical Trial Registry, up to April 5, 2019. HR and 95\% CI for overall survival (OS), cancer-specific survival (CSS), and recurrence-free survival (RFS) were calculated to estimate the effect size.

Results: A total of 18 studies with 9,400 patients were included. Overall, a high level of pretreatment AST/ALT ratio was significantly associated with worse OS (pooled HR=1.70, 95\% CI $=1.38-2.09$ ). The statistical significance was observed in all cancer types, including renal cell carcinoma (pooled $\mathrm{HR}=1.64,95 \% \mathrm{CI}=1.30-2.05$ ), liver cancer (pooled $\mathrm{HR}=1.16$, 95\% $\mathrm{CI}=1.04-1.29$ ), urinary tract urothelial carcinoma (pooled $\mathrm{HR}=1.96,95 \%$ $\mathrm{CI}=1.53-2.51$ ), bladder cancer (pooled $\mathrm{HR}=2.66,95 \% \mathrm{CI}=1.69-4.20$ ), and other cancers (pooled $\mathrm{HR}=1.44,95 \% \mathrm{CI}=1.18-1.76$ ). Moreover, an increased level of serum AST/ALT ratio predicted unfavorable $\mathrm{CSS}$ (pooled $\mathrm{HR}=2.07$, 95\% $\mathrm{CI}=1.74-2.46$ ) and $\mathrm{RFS}$ (pooled $\mathrm{HR}=1.51,95 \% \mathrm{CI}=1.15-1.99)$.

Conclusion: Elevated level of serum AST/ALT ratio before treatment is significantly associated with poor clinical outcomes of OS, CSS, and RFS in patients with solid tumors. Pretreatment AST/ALT ratio can serve as a useful prognostic predictor for malignant patients.

Keywords: aspartate transaminase to alanine transaminase ratio, prognosis, solid tumor, meta-analysis

\section{Introduction}

Cancer accounts for the majority of global deaths due to its rapidly growing incidence and mortality. Moreover, cancer is one of the most important barriers to prolonging life expectancy in most countries in the twenty-first century. ${ }^{1}$ Different types of malignancies comprise distinct morphologies and behaviors, including tumorigenic properties, metastatic potential, and therapeutic response. This heterogeneity of cancer can be resolved by developing cancer biomarkers with clinical utility. ${ }^{2}$ Biomarkers are helpful for cancer diagnosis, and provide actionable information for risk stratification, optimal therapy, and prognostic 
prediction. ${ }^{3}$ However, only a handful of biomarkers, including CA125 for ovarian cancer, prostate-specific antigen for prostate cancer (PC), and CA19-9 for pancreatic cancer, are routinely used in clinical settings for specific cancer types because biomarker development is complex, time-consuming, and costly. ${ }^{4}$ Until now, a considerable proportion of cancer patients show advanced disease stages upon diagnosis, and this phenomenon gives way to complicated therapy and poor prognosis. Therefore, identifying clinically applicable and effective pan-cancer biomarkers for the detection and prognosis of cancer is necessary.

Considering that aspartate transaminase (AST) and alanine transaminase (ALT) are the main circulating enzymes in vivo, their serum levels not only represent hepatocellular damage or death, but also have significant prognostic roles in several malignancies, such as pancreatic and breast cancers. ${ }^{5,6}$ The ratio of serum activities of AST and ALT (AST/ALT ratio), also termed as De Ritis ratio, was first reported in 1957 as a useful indicator for hepatitis etiology, ${ }^{7}$ and is the only enzyme ratio for predicting viral hepatitis that has stood the test of time. ${ }^{8}$ Nowadays, the AST/ALT ratio is accepted as a good predictor of liver function damage, and is widely used for evaluating various causes of liver disease. Moreover, the AST/ALT ratio is closely correlated with several non-hepatic diseases, such as type 2 diabetes mellitus, peripheral arterial occlusive disease, acute ischemic stroke, and esophageal cancer. ${ }^{9-12}$ Furthermore, the De Ritis ratio is closely associated with markers for impaired renal function (eGFR), cardiac strain (NTproBNP), and inflammation (CRP), suggesting that it could serve as a surrogate marker for end-organ damage in impaired systemic perfusion. ${ }^{13}$

AST and ALT production might be influenced by cancerrelated changes in metabolism, and pretreatment AST/ALT ratio is closely associated with the survival outcomes of patients with various carcinomas. ${ }^{14}$ However, these results are conflicting due to variance in study designs and sample sizes, resulting in the unknown prognostic role of AST/ALT ratio in patients with solid tumors. Therefore, we searched for available studies and conducted this first meta-analysis to reveal the prognostic value of AST/ALT ratio in solid tumors.

\section{Materials and methods}

\section{Literature search}

This meta-analysis was conducted in accordance with the Preferred Reporting Items for Systematic Reviews and
Meta-Analyses (PRISMA). ${ }^{15}$ This study was prospective registered in PROSPERO (http://www.crd.york.ac.uk/ PROSPERO/; CRD42019122772).

A comprehensive electronic search was performed based on PubMed, EMBASE, Web of Science, Cochrane Library, and Wanfang databases without any language restrictions. The databases of trial registries, including ClinicalTrials.gov, WHO International Clinical Trials Registry Platform, and Chinese Clinical Trial Registry (ChiCTR.org.cn), were also checked for eligible studies. The following search terms were used: (((“Aspartate Aminotransaminase"[All Fields] OR “AST" [All Fields]), OR ("Alanine Transaminase" [All Fields] OR "ALT" [All Fields]) OR ("De Ritis ratio" [All Fields])), AND ("Neoplasms" [All Fields] OR "Tumor" [All Fields] OR "Cancer" [All Fields] OR "Malignancy" [All Fields] OR "Carcinoma" [All Fields]), AND ("Prognosis" [All Fields] OR "Survival" [All Fields] OR "Outcome" [All Fields] OR "Mortality" [All Fields])). The last search was updated on April 5, 2019. In order to widen the literature search, the reference lists of the retrieved studies were also screened manually to identify potentially relevant studies.

\section{Study selection}

The literature search, study selection, and validation were systematically carried out by two independent reviewers (LC and WKT) who were blinded to the authors and publication years. Reviewers addressed disagreements by discussion, and the arbitrator (JYW) adjudicated any unsolved disagreement. Studies were considered eligible in this meta-analysis according to the following criteria: 1) cohort studies reported the association between AST/ALT ratio and prognosis of non-hematologic solid tumors; 2) serum AST/ALT ratio was measured before any treatment; 3) publications provided sufficient information to extract or calculate HR estimations; and 4) full-text articles. The following studies were excluded: 1) abstracts, letters, reviews, editorials, case reports, basic studies, or nonhuman studies; 2) studies with insufficient data for the estimation of HRs and $95 \%$ CIs; and 3) overlapping or duplicate studies.

\section{Data extraction and quality assessment}

The data extraction and quality assessment were independently performed by two reviewers (ZH and WKT) using a standardized extraction form. Discrepancies were settled by discussion and consensuses were reached in all cases. 
The following information was recorded from each eligible study: name of first author, year of publication, study region, duration time, cancer type, clinical stage, primary treatment, follow-up time, sample size, cutoff value, case number with elevated AST/ALT ratio, analysis method, survival outcomes, HR estimation, and quality scores. Overall survival (OS), cancer-specific survival (CSS), and recurrence-free survival (RFS) were analyzed. HR estimation was extracted from multivariate analyses when available. Otherwise, it was obtained from univariate analysis or calculated from the Kaplan-Meier curves.

The quality of each included study was systematically evaluated using the Newcastle-Ottawa Scale (NOS) for cohort studies. The total score of NOS ranged from 0 to 9 , and studies with scores $\geq 7$ were defined as high-quality.

\section{Statistical analysis}

STATA 14.0 (STATA Corporation, College Station, TX, USA) was used for all statistical analyses. Pooled HRs and 95\% CIs were used to assess the strength of pretreatment AST/ALT ratio with survival endpoints. $\mathrm{HR}>1$ denoted a worse prognosis in patients with elevated serum AST/ALT ratio, and the result was statistically significant if the $95 \%$ CI did not overlap 1. The significance of the pooled HR was determined by using $Z$-test, of which the results were statistically significant if $P<0.05$. The inter-study heterogeneity was examined by using Cochran's $Q$ test and Higgins $I^{2}$ metric. If $P<0.05$ or $I^{2}>50 \%$, a random-effects model (DerSimonianand Laird method) was applied to calculate the pooled HRs because of the possibility of substantial heterogeneity. Otherwise, a fixedeffect model was utilized (Mantel-Haenszel method) in the data synthesis. Subgroup and meta-regression analyses were performed to explore the potential factors for heterogeneity. Sensitivity analysis was conducted using a "one-study removed" model to assess the stability of the overall results. Potential publication bias was assessed by using funnel plots visually, whose results were confirmed by using Begg's and Egger's tests. The trim-and-fill method was performed to estimate an adjusted effect size if significant publication bias was identified. ${ }^{16}$ All the statistical tests were two-tailed, and $P<0.05$ was considered statistically significant.

\section{Results}

\section{Study characteristics}

A total of 328 individual studies were identified through an initial literature search. Afterward, 219 studies were excluded due to obvious irrelevance. After the titles and abstracts were screened, 66 studies that did not meet the inclusion criteria were removed. A total of 25 publications were excluded from the remaining 43 potential studies after full-text evaluation, including 5 without survival analysis, 6 without sufficient information for HR estimation, 2 with other survival outcome reports, and 12 without AST/ALT ratio data. Ultimately, 18 studies were included in the meta-analysis ${ }^{17-34}$ (Figure 1).

The basic characteristics of the included studies are summarized in Table 1. The 18 included studies published between 2011 and 2019 provided an overall sample size of 9,400 , with a range of 74-2,965. The six studies were carried out in People's Republic of China (including Taiwan), ${ }^{21,22,24,26,33,34}$ followed by Japan $(n=4),{ }^{20,23,28,31}$ Korea $(n=5),{ }^{17,18,27,29,30}$ Turkey $(n=2),{ }^{19,25}$ and the USA $(n=1) .{ }^{32}$ Regarding cancer type, 6 studies reported renal cell cancer (RCC), ${ }^{18,19,22,23,30,32} 4$ reported liver cancer with hepatocellular carcinoma (HCC), ${ }^{21,26,33,34} 3$ reported urinary tract urothelial carcinoma (UTUC), ${ }^{27-29} 2$ reported PC, ${ }^{20,24} 2$ reported bladder cancer, ${ }^{17,25}$ and 1 reported head and neck cancer. ${ }^{31}$ Considering survival endpoint, 16 studies focused on $\mathrm{OS},{ }^{17-23,25-27,29-34} 8$ focused on $\mathrm{CSS},{ }^{17-19,23,25,27,29,30}$ and 5 focused on RFS. ${ }^{24,27,28,30,34}$ All the 18 studies were written in English. The HR estimations in the 14 studies were directly extracted from multivariate analyses, and those of the other 4 studies were obtained from univariate analyses. ${ }^{19,20,26,34}$ All included studies had a score of 7 or more and were of high quality based on the NOS criteria.

\section{Primary outcome: OS}

A total of 16 studies with 8,853 enrolled patients provided sufficient data for OS (Table 2). The overall outcome demonstrated that increased pretreatment AST/ALT ratio was significantly associated with worse OS (pooled $\mathrm{HR}=1.70,95 \% \mathrm{CI}=1.37-2.09, P<0.001$, random effects, Figure 2) with significant heterogeneity across studies $\left(I^{2}=85.4 \%, P_{\mathrm{h}}<0.001\right)$. A high $\mathrm{AST} / \mathrm{ALT}$ ratio was related to the decreased OS in patients with $\mathrm{RCC}$ (pooled $\mathrm{HR}=1.64$, 95\% CI $=1.30-2.05, P<0.001$, random effects), liver cancer (pooled $\mathrm{HR}=1.16,95 \% \mathrm{CI}=1.04-1.29, P=0.006$, random effects), UTUC (pooled $\mathrm{HR}=1.96,95 \% \mathrm{CI}=1.53-2.51$, $P<0.001$, fixed effects), bladder cancer (pooled $\mathrm{HR}=2.66$, $95 \% \mathrm{CI}=1.69-4.20, P<0.001$, fixed effects), and other cancers (pooled $\mathrm{HR}=1.44,95 \% \mathrm{CI}=1.18-1.76, P=0.001$, fixed effects) when the subgroup analyses were conducted according to various cancer types. A significant relationship of elevated AST/ALT ratio and poor OS was found in patients that underwent surgery (pooled $H R=1.74,95 \%$ 


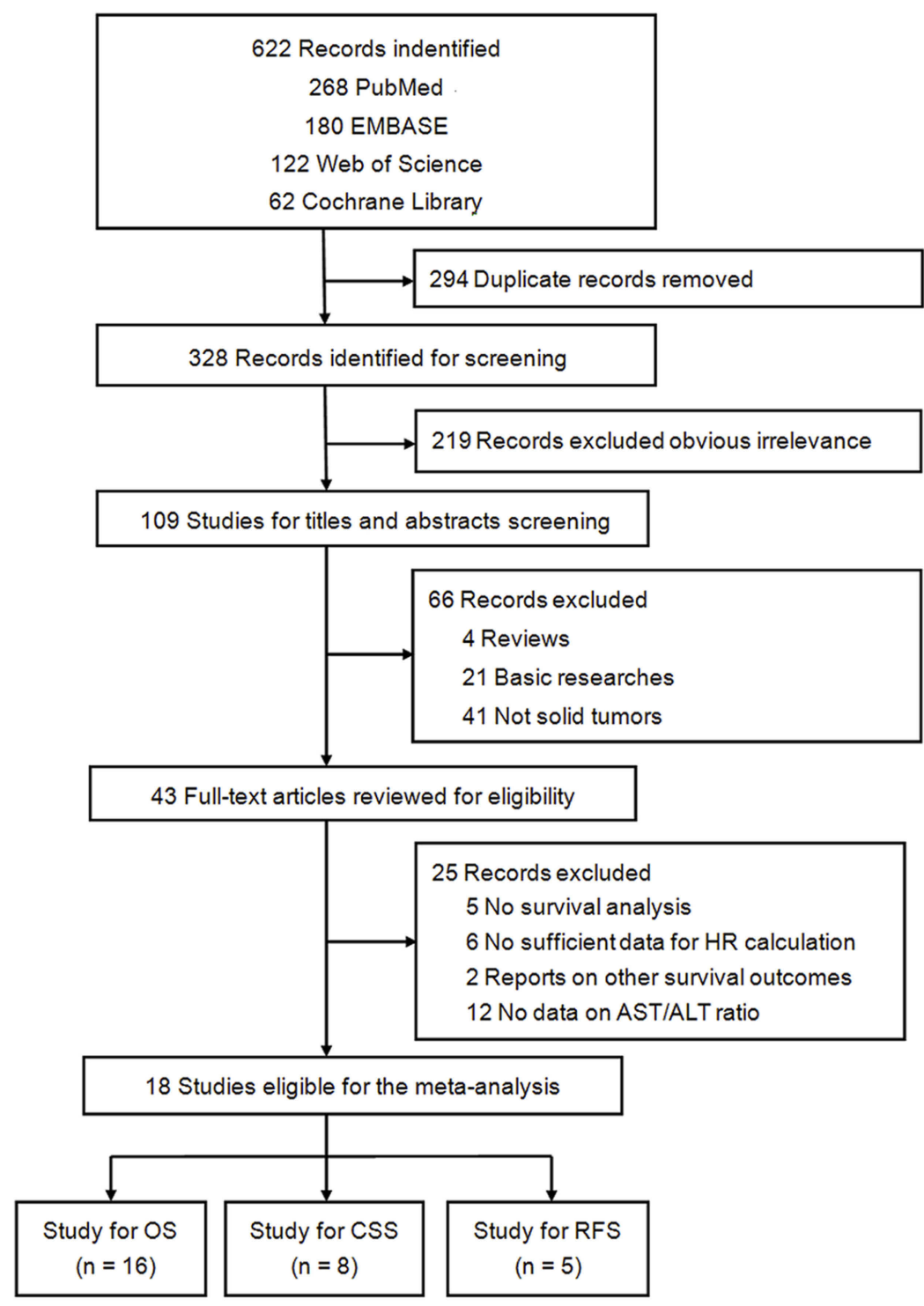

Figure I Flow diagram of the study selection process.

Abbreviations: AST/ALT ratio, aspartate transaminase to alanine transaminase ratio; OS, overall survival; CSS, cancer-specific survival; RFS, recurrence-free survival.

$\mathrm{CI}=1.34-2.25, \quad P<0.001$, random effects) and did not undergo surgery (pooled $\mathrm{HR}=1.50,95 \% \mathrm{CI}=1.26-1.78$, $P<0.001$, fixed effects) upon stratification by the primary treatment. Additionally, the overall results did not show obvious alterations when the included studies were grouped on the basis of study population, clinical stage, cutoff value, analysis method, and sample size.

\section{Secondary outcome: CSS and RFS}

Eight cohorts with 5,640 patients reported the CSS outcome (Table 3), and the pooled outcome suggested that a high level of pretreatment AST/ALT ratio was significantly associated with short CSS (pooled $\mathrm{HR}=2.07,95 \% \mathrm{CI}=1.74-2.46$, $P<0.001$, fixed effects, Figure $3 \mathrm{~A}$ ) with insignificant heterogeneity $\left(I^{2}=38.1 \%, P_{\mathrm{h}}=0.126\right)$. An elevated AST/ALT ratio predicted poor CSS for patients with RCC (pooled $\mathrm{HR}=1.78,95 \% \mathrm{CI}=1.42-2.22, P<0.001$, fixed effects), bladder cancer (pooled $\mathrm{HR}=3.78,95 \% \mathrm{CI}=2.02-7.04, P<0.001$, fixed effects), and UTUC (pooled $\mathrm{HR}=2.38,95 \% \mathrm{CI}=1.76-3.22$, $P<0.001$, fixed effects) when subgroup analysis was conducted based on cancer type. These positive results did not change when the subgroup analyses of clinical stage, cutoff value, and sample size were conducted. However, the positive correlation of the increased AST/ALT ratio and poor CSS existed in the Asian (pooled $\mathrm{HR}=2.12,95 \% \mathrm{CI}=1.76-2.56, P<0.001$, fixed effects) but not in the Caucasian studies (pooled $\mathrm{HR}=2.60$, $95 \%$ CI $=0.61-11.09, P=0.198$, random effects) upon study population stratification. 


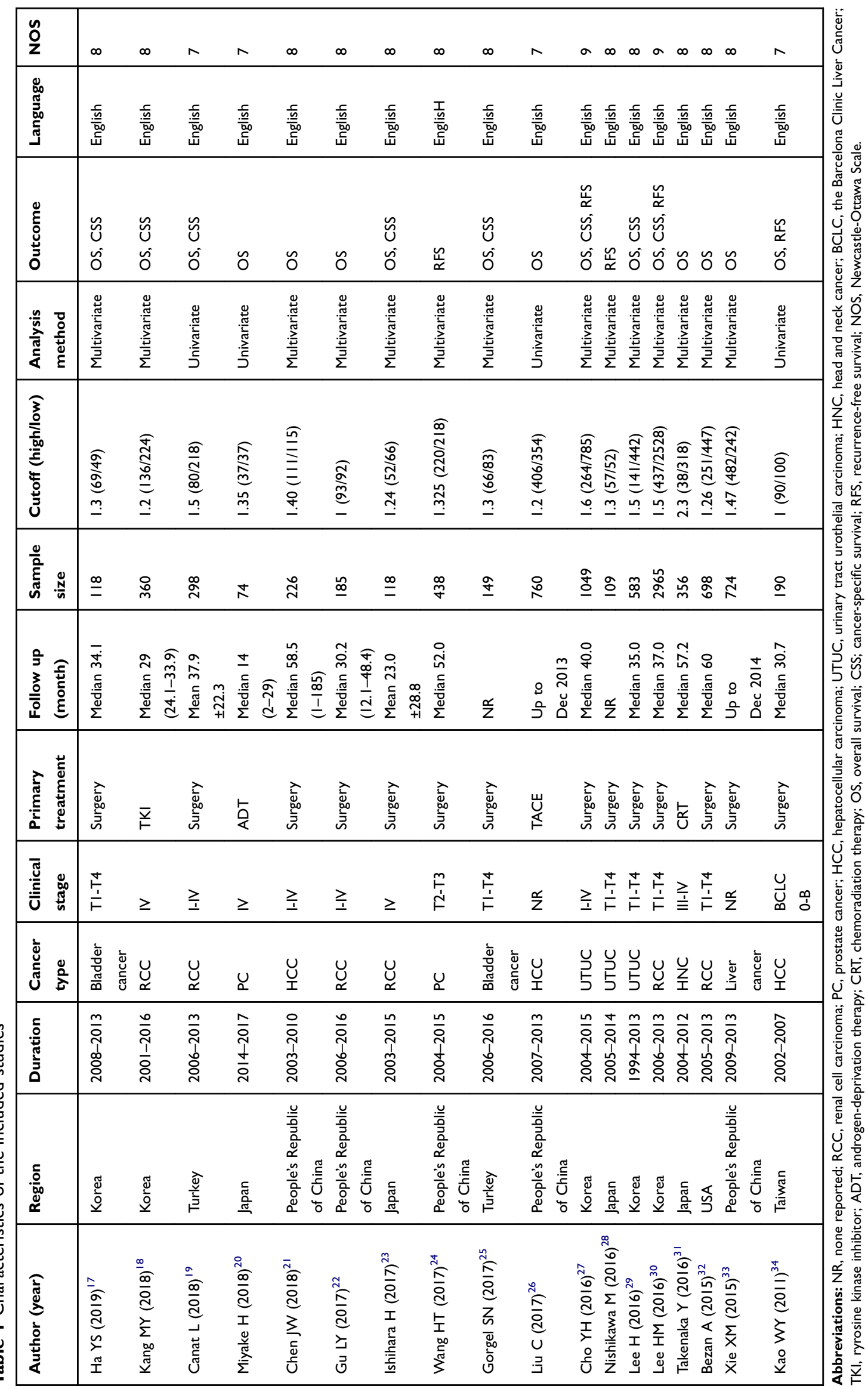


Table 2 Pooled and subgroup analyses of data synthesis of overall survival for this meta-analysis

\begin{tabular}{|c|c|c|c|c|c|c|c|c|}
\hline \multirow[t]{2}{*}{ Categories } & \multirow[t]{2}{*}{ Study (case) } & \multirow[t]{2}{*}{ Model } & \multirow[t]{2}{*}{ HR (95\% Cl) } & \multirow[t]{2}{*}{$\mathbf{Z}$} & \multirow[t]{2}{*}{$P_{\mathrm{z}}$} & \multicolumn{2}{|c|}{ Heterogeneity } & \multirow[t]{2}{*}{$P_{m}$} \\
\hline & & & & & & $I^{2}$ & $\boldsymbol{P}_{\mathrm{h}}$ & \\
\hline Overall survival (OS) & $16(8853)$ & Random & $1.70(1.37-2.09)$ & 4.96 & $<0.001$ & $85.4 \%$ & $<0.001$ & \\
\hline $\begin{array}{l}\text { Study population } \\
\text { Asian } \\
\text { Caucasian }\end{array}$ & $\begin{array}{l}\text { I3 (7708) } \\
3(1145)\end{array}$ & $\begin{array}{l}\text { Random } \\
\text { Random }\end{array}$ & $\begin{array}{l}1.73(1.35-2.22) \\
1.59(1.05-2.42)\end{array}$ & $\begin{array}{l}4.32 \\
2.17\end{array}$ & $\begin{array}{l}<0.001 \\
0.030\end{array}$ & $\begin{array}{l}87.1 \% \\
79.4 \%\end{array}$ & $\begin{array}{l}<0.001 \\
0.008\end{array}$ & 0.105 \\
\hline $\begin{array}{l}\text { Cancer type } \\
\text { RCC } \\
\text { Liver cancer } \\
\text { UTUC } \\
\text { Bladder cancer } \\
\text { Others }\end{array}$ & $\begin{array}{l}6(4624) \\
4(1900) \\
2(1632) \\
2(267) \\
2(430)\end{array}$ & $\begin{array}{l}\text { Random } \\
\text { Random } \\
\text { Fixed } \\
\text { Fixed } \\
\text { Fixed }\end{array}$ & $\begin{array}{l}1.64(1.30-2.05) \\
1.16(1.04-1.29) \\
1.96(1.53-2.5 I) \\
2.66(1.69-4.20) \\
1.44(1.18-1.76)\end{array}$ & $\begin{array}{l}4.26 \\
2.75 \\
5.31 \\
4.21 \\
3.62\end{array}$ & $\begin{array}{l}<0.001 \\
0.006 \\
<0.001 \\
<0.001 \\
<0.001\end{array}$ & $\begin{array}{l}55.6 \% \\
95.1 \% \\
0.0 \% \\
0.0 \% \\
26.8 \%\end{array}$ & $\begin{array}{l}0.046 \\
<0.001 \\
0.538 \\
0.910 \\
0.242\end{array}$ & 0.090 \\
\hline $\begin{array}{l}\text { Primary treatment } \\
\text { Surgery } \\
\text { None surgery }\end{array}$ & $\begin{array}{l}\text { I3 (8063) } \\
3(790)\end{array}$ & $\begin{array}{l}\text { Random } \\
\text { Fixed }\end{array}$ & $\begin{array}{l}1.74(1.34-2.25) \\
1.50(1.26-1.78)\end{array}$ & $\begin{array}{l}4.21 \\
4.61\end{array}$ & $\begin{array}{l}<0.001 \\
<0.001\end{array}$ & $\begin{array}{l}88.0 \% \\
0.0 \%\end{array}$ & $\begin{array}{l}<0.001 \\
0.374\end{array}$ & 0.783 \\
\hline $\begin{array}{l}\text { Clinical stage } \\
\text { I-IV } \\
\text { TI-T4 } \\
\text { IV } \\
\text { Others }\end{array}$ & $\begin{array}{l}4(1758) \\
5(45 \mid 3) \\
3(552) \\
4(2030)\end{array}$ & $\begin{array}{l}\text { Random } \\
\text { Fixed } \\
\text { Fixed } \\
\text { Random }\end{array}$ & $\begin{array}{l}1.65(1.15-2.35) \\
1.89(1.59-2.25) \\
1.83(1.43-2.34) \\
I .17(1.06-1.29)\end{array}$ & $\begin{array}{l}2.74 \\
7.26 \\
4.79 \\
3.11\end{array}$ & $\begin{array}{l}0.006 \\
<0.001 \\
<0.001 \\
0.003\end{array}$ & $\begin{array}{l}70.5 \% \\
0.0 \% \\
0.0 \% \\
94.9 \%\end{array}$ & $\begin{array}{l}0.017 \\
0.450 \\
0.695 \\
<0.001\end{array}$ & 0.111 \\
\hline $\begin{array}{l}\text { Cutoff value } \\
>1.3 \\
\leq 1.3\end{array}$ & $\begin{array}{l}8(6275) \\
8(2578)\end{array}$ & $\begin{array}{l}\text { Random } \\
\text { Fixed }\end{array}$ & $\begin{array}{l}\text { I.48 (I.08-2.02) } \\
\text { I.78 (1.59-2.00) }\end{array}$ & $\begin{array}{l}2.44 \\
9.79\end{array}$ & $\begin{array}{l}0.015 \\
<0.001\end{array}$ & $\begin{array}{l}89.4 \% \\
0.0 \%\end{array}$ & $\begin{array}{l}<0.001 \\
0.550\end{array}$ & 0.114 \\
\hline $\begin{array}{l}\text { Analysis method } \\
\text { Univariate } \\
\text { Multivariate }\end{array}$ & $\begin{array}{l}4(1322) \\
12(7531)\end{array}$ & $\begin{array}{l}\text { Random } \\
\text { Random }\end{array}$ & $\begin{array}{l}1.56(1.20-2.04) \\
1.76(1.33-2.33)\end{array}$ & $\begin{array}{l}3.32 \\
3.95\end{array}$ & $\begin{array}{l}0.001 \\
<0.001\end{array}$ & $\begin{array}{l}68.8 \% \\
87.6 \%\end{array}$ & $\begin{array}{l}0.022 \\
<0.001\end{array}$ & 0.890 \\
\hline $\begin{array}{l}\text { Sample size } \\
\quad \geq 200 \\
\quad<200\end{array}$ & $\begin{array}{l}10(8019) \\
6(834)\end{array}$ & $\begin{array}{l}\text { Random } \\
\text { Fixed }\end{array}$ & $\begin{array}{l}1.50(1.17-1.92) \\
2.18(1.75-2.72)\end{array}$ & $\begin{array}{l}3.20 \\
6.96\end{array}$ & $\begin{array}{l}0.001 \\
<0.001\end{array}$ & $\begin{array}{l}89.1 \% \\
0.0 \%\end{array}$ & $\begin{array}{l}<0.001 \\
0.899\end{array}$ & 0.586 \\
\hline
\end{tabular}

Notes: $P_{\mathrm{Z}}$ denotes $P$-value for statistical significance based on $Z$ test; $P_{\mathrm{h}}$ denotes $P$-value for heterogeneity based on $Q$ test; $P_{\mathrm{m}}$ denotes $P$-value for statistical outcome based on multivariate meta-regression analysis.

Abbreviations: RCC, renal cell carcinoma; UTUC, urinary tract urothelial carcinoma.

Five studies comprising 4,751 cases provided the data for the RFS endpoint (Table 3). A random-effects model was applied to calculate the pooled result due to the significant heterogeneity $\left(I^{2}=64.2 \%, P_{\mathrm{h}}=0.025\right)$, which indicated that elevated AST/ALT ratio was obviously correlated with poor RFS (pooled $\mathrm{HR}=1.51$, 95\% $\mathrm{CI}=1.15-1.99, P=0.003$, random effects, Figure 3B).

\section{Sensitivity analysis and meta-regression analysis}

The results of sensitivity analysis suggested that the conclusions for OS, CSS, and RFS were stable because the observed effect size (the pooled HRs) was not significantly affected by the exclusion of any individual study (Figure 4).

We performed meta-regression analyses to investigate the suspected causes of heterogeneity among studies for each survival outcome. The results demonstrated that study population $(P=0.105)$, cancer type $(P=0.090)$, primary treatment $(P=0.783)$, clinical stage $(P=0.111)$, cutoff value $(P=0.114)$, analysis method $(P=0.890)$, and sample size $(P=0.586)$ have no effects on the heterogeneity for OS (Table 2). Moreover, study population $(P=0.893)$, cancer type $(P=0.574)$, clinical stage $(P=0.536)$, cutoff value $(P=0.316)$, and sample size $(P=0.199)$ did not contribute to the source of heterogeneity for CSS (Table 3 ). 


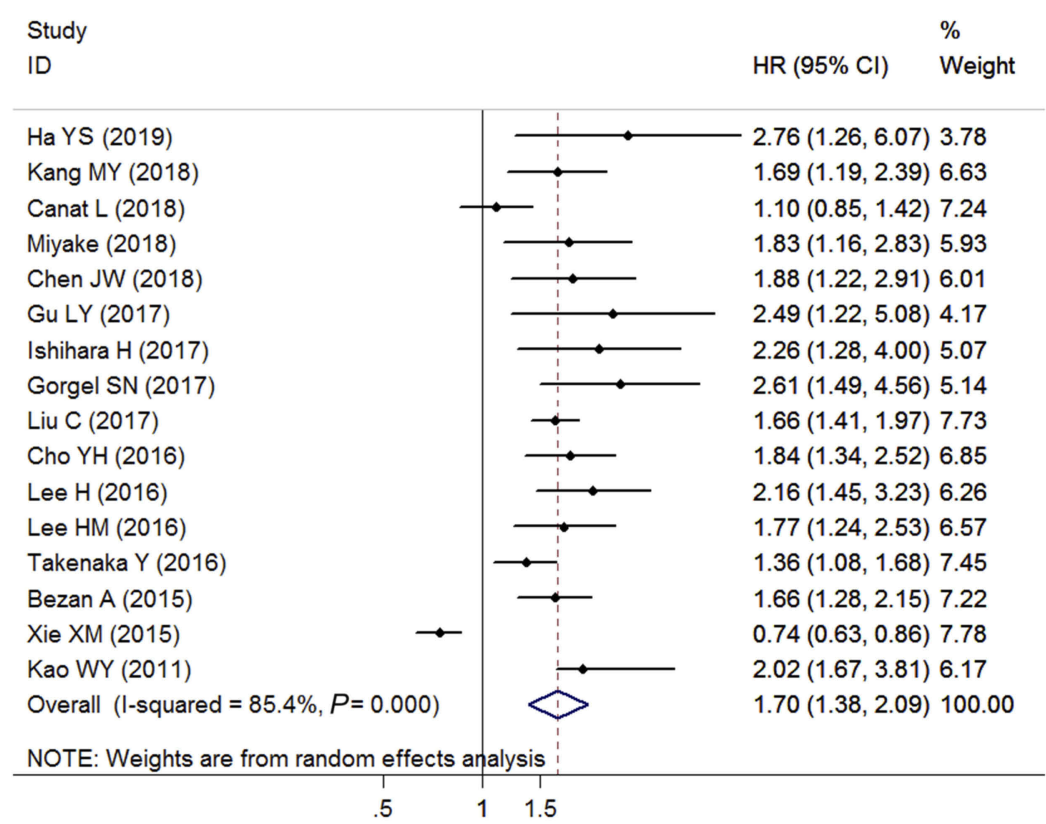

Figure 2 Forest plots of the association between AST/ALT ratio and overall survival. Abbreviation: AST/ALT ratio, aspartate transaminase to alanine transaminase ratio.

Table 3 Pooled and subgroup analyses of data synthesis of cancer-specific survival and recurrence-free survival for this meta-analysis

\begin{tabular}{|c|c|c|c|c|c|c|c|c|}
\hline \multirow[t]{2}{*}{ Categories } & \multirow[t]{2}{*}{ Study (case) } & \multirow[t]{2}{*}{ Model } & \multirow[t]{2}{*}{ HR $(95 \% \mathrm{Cl})$} & \multirow[t]{2}{*}{$\mathbf{Z}$} & \multirow[t]{2}{*}{$P_{\mathbf{z}}$} & \multicolumn{2}{|c|}{ Heterogeneity } & \multirow[t]{2}{*}{$P_{m}$} \\
\hline & & & & & & $I^{2}$ & $P_{h}$ & \\
\hline Cancer-specific survival (CSS) & $8(5640)$ & Fixed & $2.07(1.74-2.46)$ & 8.25 & $<0.001$ & $38.1 \%$ & 0.126 & \\
\hline $\begin{array}{l}\text { Study population } \\
\text { Asian } \\
\text { Caucasian }\end{array}$ & $\begin{array}{l}6(5193) \\
2(447)\end{array}$ & $\begin{array}{l}\text { Fixed } \\
\text { Random }\end{array}$ & $\begin{array}{l}2.12(1.76-2.56) \\
2.60(0.61-11.09)\end{array}$ & $\begin{array}{l}7.86 \\
1.29\end{array}$ & $\begin{array}{l}<0.001 \\
0.198\end{array}$ & $\begin{array}{l}0.0 \% \\
86.2 \%\end{array}$ & $\begin{array}{l}0.605 \\
0.007\end{array}$ & 0.893 \\
\hline $\begin{array}{l}\text { Cancer type } \\
\text { RCC } \\
\text { Bladder cancer } \\
\text { UTUC }\end{array}$ & $\begin{array}{l}4(374 I) \\
2(267) \\
2(1632)\end{array}$ & $\begin{array}{l}\text { Fixed } \\
\text { Fixed } \\
\text { Fixed }\end{array}$ & $\begin{array}{l}1.78(1.42-2.22) \\
3.78(2.02-7.04) \\
2.38(1.76-3.22)\end{array}$ & $\begin{array}{l}5.01 \\
4.17 \\
5.63\end{array}$ & $\begin{array}{l}<0.001 \\
<0.001 \\
<0.001\end{array}$ & $\begin{array}{l}18.0 \% \\
24.4 \% \\
0.0 \%\end{array}$ & $\begin{array}{l}0.301 \\
0.250 \\
0.697\end{array}$ & 0.574 \\
\hline $\begin{array}{l}\text { Clinical stage } \\
\text { I-IV } \\
\text { TI-T4 } \\
\text { IV }\end{array}$ & $\begin{array}{l}2(1347) \\
4(38 \mid 5) \\
2(478)\end{array}$ & $\begin{array}{l}\text { Random } \\
\text { Fixed } \\
\text { Fixed }\end{array}$ & $\begin{array}{l}1.85(1.05-3.48) \\
2.55(1.90-3.45) \\
1.74(1.28-2.34)\end{array}$ & $\begin{array}{l}2.10 \\
6.30 \\
3.53\end{array}$ & $\begin{array}{l}0.046 \\
<0.001 \\
<0.001\end{array}$ & $\begin{array}{l}75.0 \% \\
9.5 \% \\
0.0 \%\end{array}$ & $\begin{array}{l}0.045 \\
0.345 \\
0.416\end{array}$ & 0.536 \\
\hline $\begin{array}{l}\text { Cutoff value } \\
>1.3 \\
\leq 1.3\end{array}$ & $\begin{array}{l}5(5044) \\
3(596)\end{array}$ & $\begin{array}{l}\text { Random } \\
\text { Fixed }\end{array}$ & $\begin{array}{l}2.26(1.63-3.15) \\
1.84(1.38-2.45)\end{array}$ & $\begin{array}{l}4.83 \\
4.16\end{array}$ & $\begin{array}{l}<0.001 \\
<0.001\end{array}$ & $\begin{array}{l}53.0 \% \\
0.0 \%\end{array}$ & $\begin{array}{l}0.074 \\
0.419\end{array}$ & 0.316 \\
\hline $\begin{array}{l}\text { Sample size } \\
\quad \geq 200 \\
<200\end{array}$ & $\begin{array}{l}5(5255) \\
3(385)\end{array}$ & $\begin{array}{l}\text { Fixed } \\
\text { Fixed }\end{array}$ & $\begin{array}{l}1.95(1.62-2.36) \\
2.82(1.83-4.34)\end{array}$ & $\begin{array}{l}6.95 \\
4.69\end{array}$ & $\begin{array}{l}<0.001 \\
<0.001\end{array}$ & $\begin{array}{l}33.8 \% \\
32.1 \%\end{array}$ & $\begin{array}{l}0.196 \\
0.229\end{array}$ & 0.199 \\
\hline Recurrence-free survival (RFS) & $5(475 I)$ & Random & $1.51(1.15-1.99)$ & 2.95 & 0.003 & $64.2 \%$ & 0.025 & \\
\hline
\end{tabular}

Note: $P_{\mathrm{Z}}$ denotes $P$-value for statistical significance based on $Z$ test; $P_{\mathrm{h}}$ denotes $P$-value for heterogeneity based on $Q$ test; $P_{\mathrm{m}}$ denotes $P$-value for statistical outcome based on multivariate meta-regression analysis.

Abbreviations: RCC, renal cell carcinoma; UTUC, urinary tract urothelial carcinoma. 


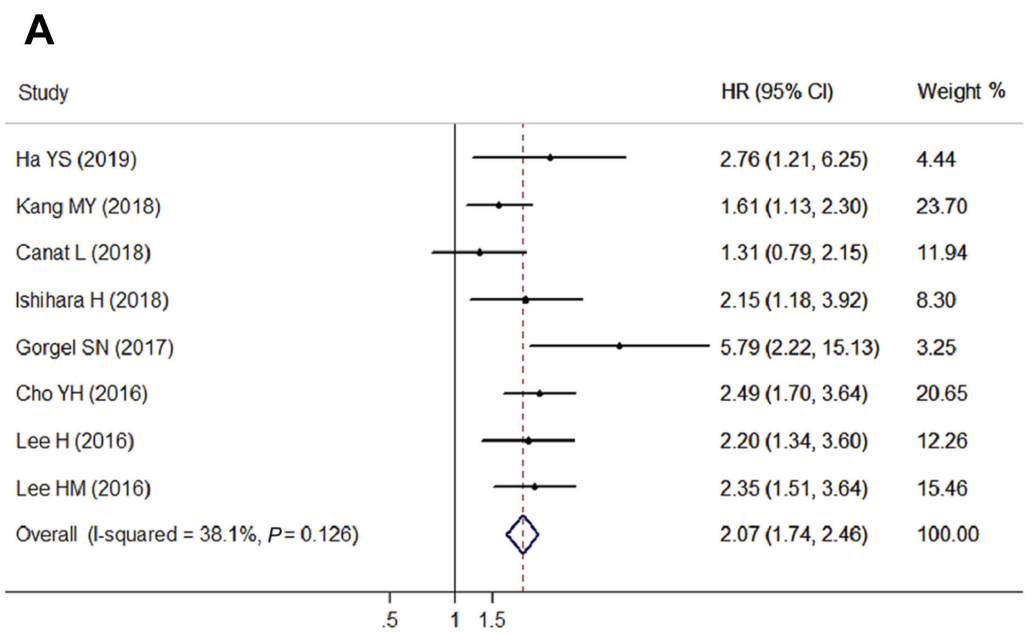

B

\begin{tabular}{|c|c|c|}
\hline Study & $\mathrm{HR}(95 \% \mathrm{Cl})$ & Weight \% \\
\hline Wang HT (2017) & $1.72(1.06,2.78)$ & 16.61 \\
\hline Cho YH (2016) & $1.20(0.98,1.46)$ & 28.96 \\
\hline Nishikawa M (2016) & $4.21(1.95,9.08)$ & 9.25 \\
\hline Lee H (2016) & $1.46(1.08,1.97)$ & 24.23 \\
\hline Kao WY (2011) & $1.24(0.85,1.79)$ & 20.96 \\
\hline Overall $(I-$ squared $=64.2 \%, P=0.025$ ) & $1.51(1.15,1.99)$ & 100.00 \\
\hline NOTE: Weights are from random effects analys/s & & \\
\hline
\end{tabular}

Figure 3 Forest plots of the association between AST/ALT ratio and (A) cancer-specific survival; (B) recurrence-free survival. Abbreviation: AST/ALT ratio, aspartate transaminase to alanine transaminase ratio.

\section{Publication bias}

Upon visual inspection of the funnel plot (Figure 5A) confirmed by Egger's test $(P=0.004)$, significant publication bias was found with regard to the pooled outcome of OS. The trim-and-fill analysis suggested that five nonpublished studies were needed to balance the funnel plot (Figure 5B). The adjusted HR and 95\% CI were attenuated but remained significant (pooled $\mathrm{HR}=1.51 ; 95 \%$ $\mathrm{CI}=1.26-1.82$; random effects), thereby suggesting that the potential publication bias had minimal impact on the overall outcome. Moreover, the funnel plots did not show unsymmetrical evidence, and neither Begg's nor Egger's test demonstrated evidence of publication bias concerning CSS (Begg's test, $P=0.536$; Egger'test, $P=0.166$; Figure 5C) and RFS (Begg's test, $P=0.186$; Egger'test, $P=0.148$; Figure 5D).

\section{Discussion}

To date, numerous studies have reported the application of aminotransaminase as a prognostic indicator in various cancers, regardless of the presence of liver-specific disease. ${ }^{35-37}$ AST is predominantly expressed in the mitochondria and is widely found in several organs, including the liver, heart, kidney, brain, and skeletal muscle, whereas ALT is only present in the hepatocyte cytoplasm. AST and ALT are the major critical enzymes in biological processes, of which their functions reflect the important link between carbohydrate and protein metabolism. The serum AST level should be higher than that of ALT due to the 2.5:1 hepatic proportion of AST/ALT. However, the resulting serum levels of AST and ALT are fairly similar due to the quicker removal rate of AST $\left(\mathrm{t}^{1} / 2=18 \mathrm{hrs}\right)$ by the liver sinusoids compared with that of ALT $\left(\mathrm{t}^{1} 1 / 2=36 \mathrm{hrs}\right)$. The 

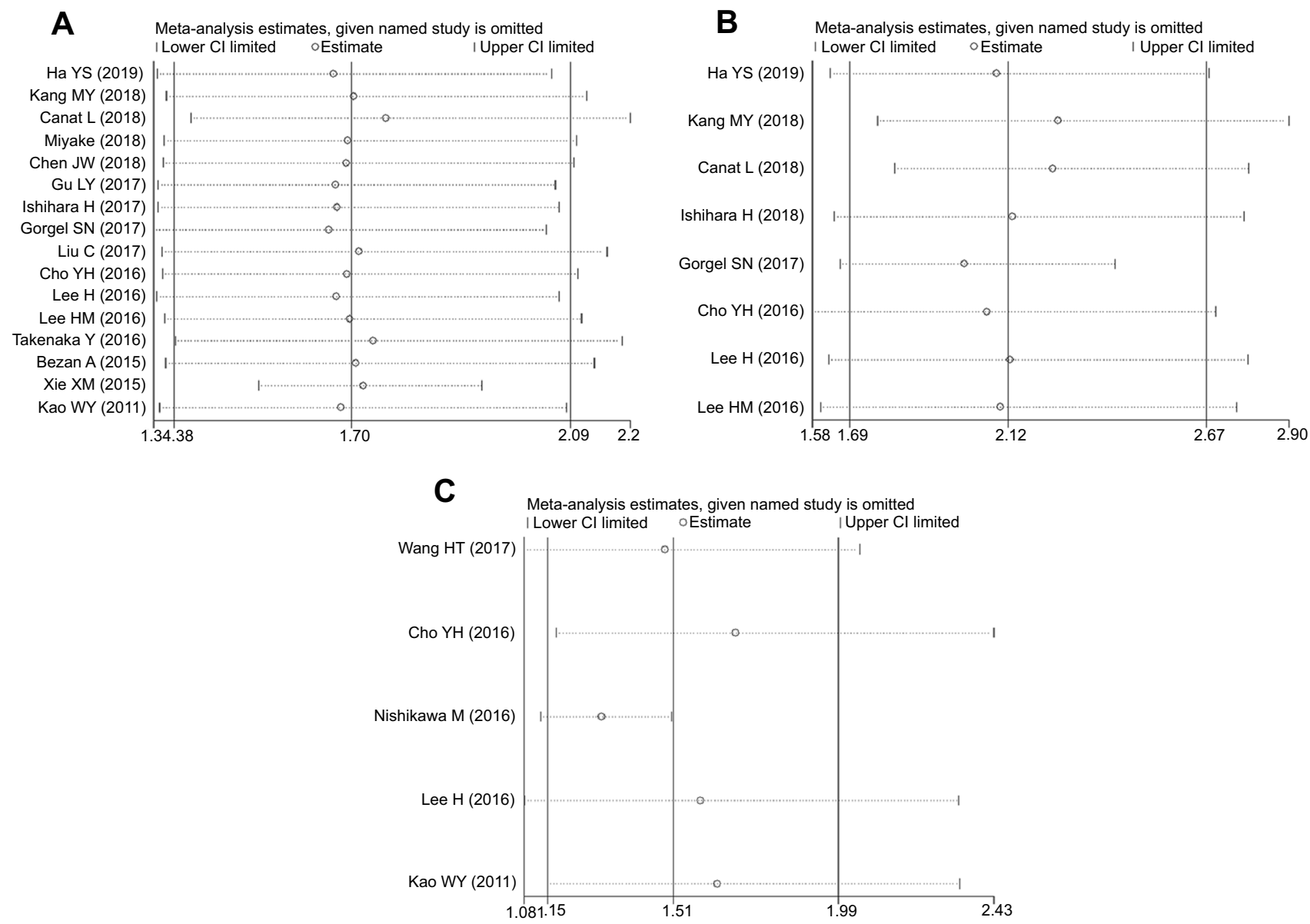

Figure 4 Sensitivity analysis for (A) overall survival; (B) cancer-specific survival; (C) recurrence-free survival.

ratio of AST and ALT is normally constant among healthy individuals. Serum AST and ALT levels, as part of "liver function tests" panel, are routinely assessed before clinical treatment. Compared with tissue biomarkers, hematological biomarkers are easier to obtain, do not involve invasive operations, and involve inexpensive operations, thus making them convenient and reproducible preoperative parameters for risk prediction. ${ }^{38}$ All laboratories that report abnormal ALT are recommended to report the patients' AST levels to allow the calculation of the De Ritis ratio, which provides valuable diagnostic and prognostic information. $^{39}$ Moreover, the serum level of AST and ALT can be influenced by many other non-tumor-related factors, including chronic hepatitis, coronary heart disease, impaired renal function, and drugs. Hence, their numerical values are unstable when they act as single predictors. Instead, the combination of AST and ALT is more appropriate as a composite parameter and can counteract the effects of other factors.
AST/ALT ratio has been frequently used as a predictive index for various diseases, including critical limb ischemia, ${ }^{10}$ arterial stiffness, ${ }^{40}$ and renal and respiratory dysfunction. ${ }^{41}$ The effect of AST/ALT ratio on cancer prognosis has been declared in several studies. However, these studies presented extremely different results. Thus, the exact value of AST/ ALT ratio on malignance prognosis remains uncertain. We conducted this first meta-analysis to comprehensively examine the association between pretreatment AST/ALT ratio and the prognosis of solid tumors. The pooled risk of increased AST/ALT ratio for worse OS in patients with malignancies was considerably with a combined HR of 1.70 (95\% CI: 1.38-2.09). Generally, in the subgroup analyses, elevated AST/ALT ratio is an important prognostic predictor in patients with cancer for OS, regardless of study population, cancer type, primary treatment, clinical stage, cutoff value, analysis method, and sample size. Furthermore, a significantly negative association was found between pretreatment AST/ALT ratio and both CSS (pooled HR=2.07, 

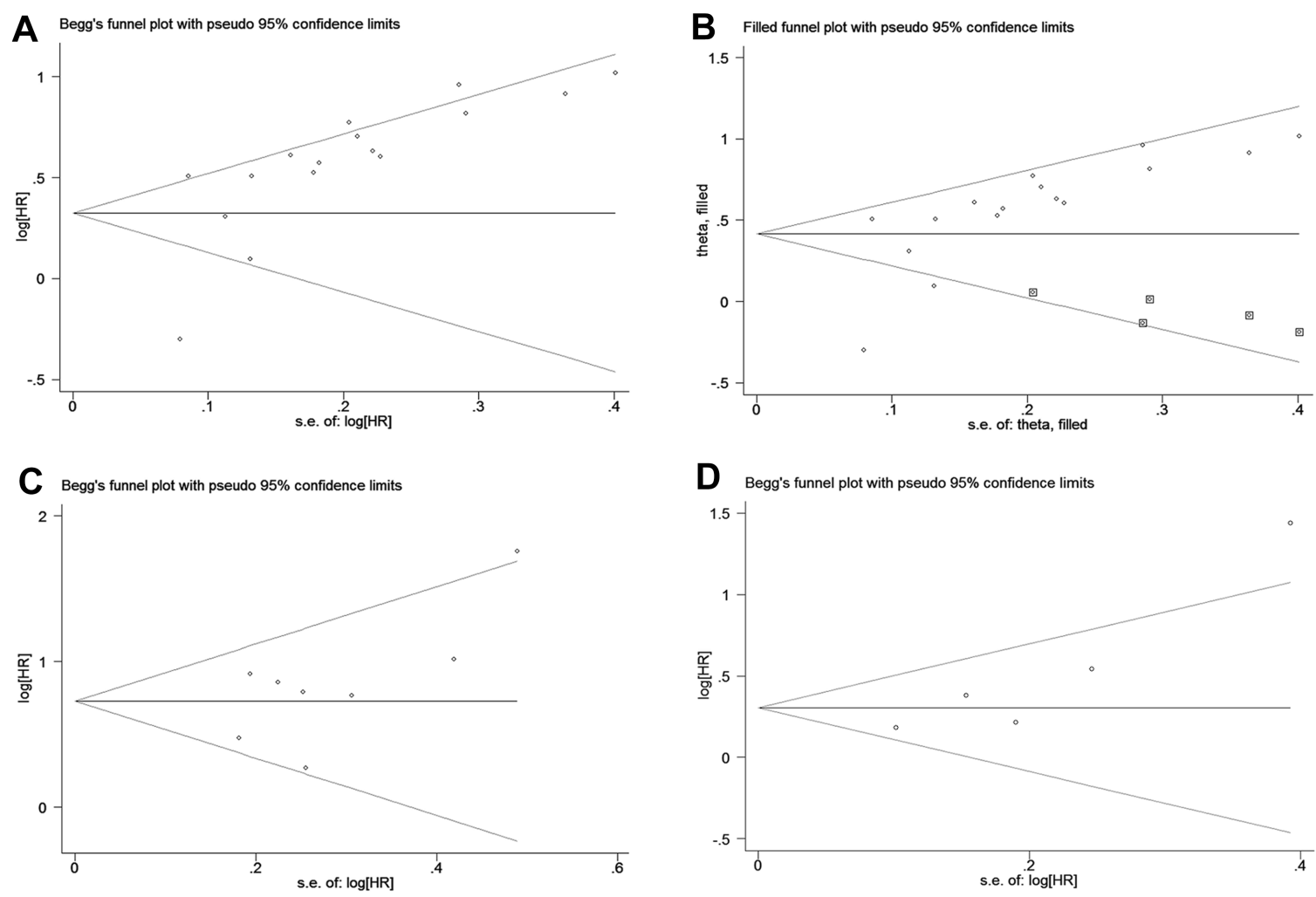

Figure 5 Funnel plots assessing the potential publication bias of the included studies. (A) Funnel plot of publication bias for overall survival. (B) Funnel plot adjusted by trim-and-fill analysis for overall survival. (C) Funnel plot of publication bias for cancer-specific survival. (D) Funnel plot adjusted by trim and fill analysis for recurrence-free survival.

95\% CI=1.74-2.46, $P<0.001$ ) and RFS (pooled $\mathrm{HR}=1.51$, 95\% CI=1.15-1.99, $P=0.003)$. Hence, the serum AST/ALT ratio before treatment may be a negative prognostic factor for malignant patients. However, the insignificant correlations of AST/ALT ratio and CSS were unveiled in the subgroup of Caucasian patients. Notably, these insignificant results were achieved based on limited studies and insufficient sample sizes. Hence, they should be interpreted with caution, and additional studies are needed to support or validate the conclusion.

Sensitivity analyses have confirmed the robustness of the pooled outcomes, but moderate to extreme inter-study heterogeneity were found for each survival endpoint, and this finding might be attributed to the different baseline characteristics of the individual studies. Thus, we employed metaregression analysis by using study population, cancer type, clinical stage, primary treatment, cutoff value, analysis method, and sample size to investigate the potential source of heterogeneity. However, none of these factors could explain the heterogeneity for OS. Similar results were achieved concerning CSS. Subgroup analyses were conducted to assess the source of heterogeneity, which was slightly reduced in most subgroups, but remained statistically significant $(P<0.05)$ in several subgroups. Instead, we calculated the effect size by using a random-effects model to minimize the effects of heterogeneity on the pooled outcome. Due to the limited number of included studies, we did not perform subgroup analysis and meta-regression analysis on RFS. Moreover, although we have performed a broad literature search, a potential publication bias was still found among the studies reporting OS, which may have inflated the pooled outcomes. After trim-and-fill analysis recalculation, the adjusted HR estimation reinforced the prognostic value of AST/ALT ratio concerning OS but remained statistically, indicating that the publication bias may have little systematic impact on the overall finding. Therefore, the results of this meta-analysis are robust and reliable.

The internal mechanisms of elevated AST/ALT ratio associated with poor outcomes of patients with cancer remain unclear. Previous studies hypothesized the involvement of glucose metabolism. Cancer cells favor a high rate of aerobic glycolysis for a high proliferative status compared with normal cells. ${ }^{42}$ AST is known to play a vital role in aerobic glycolysis by relocating nicotinamide adenine dinucleotide hydrogen into the mitochondria through malate-shuffling, as well as $\alpha$-ketoglutarate, aspartate and glutamate. ${ }^{43}$ This 
pathological condition leads to the activation of more AST than ALT in rapidly growing cancer tissues. ${ }^{44}$ Moreover, pyruvate production and glutaminolysis are enhanced in malignant cells, of which the conversion of pyruvate and glutamate to alanine and $\alpha$-ketoglutarate, respectively, is catalyzed by ALT. $^{45}$ ALT level is lower in highly invasive cells than in less invasive cells to meet the demand for tumor cell metabolism. ${ }^{46}$ The major cancer types involved in our meta-analysis, including RCC, UTUC, PC, bladder cancer, and $\mathrm{HCC}$, are known as glucose-dependent malignancies. Thus, the AST/ALT ratio may reflect the metabolic alterations in these glucose-utilizing cancers, which are possibly related to tumor growth and progression. Furthermore, oxidative stress and inflammation are closely related to cancer development. $^{47,48}$ Simultaneously, oxidative stress and inflammation can cause liver injury. ${ }^{49}$ The changes in serum ALT and AST levels have become common diagnostic markers for assessing hepatic function. ${ }^{50}$ Thus, the elevated AST/ALT ratio represents high oxidative stress and inflammatory environment in vivo that are linked to the poor survival of patients with different cancer types.

Some potential limitations should be acknowledged in the present meta-analysis. First, heterogeneity may result from the inconsistencies in the cutoff values and follow-up periods among the individual studies. Second, several individual HR estimations were obtained from univariate analyses or survival curves, which have not adjusted the confounding factors, leading to less reliability than those HRs that were directly calculated from multivariate analyses. Third, the included studies were mainly conducted in East Asian countries, of which the racial representation was limited; thus, conclusions should be cautiously applied for other racial populations. Fourth, this meta-analysis was limited to published literature, which represents positive results over negative findings, resulting in an overestimation of the overall outcomes to some extent. The enrolled studies provided low-quality data with short follow-up period. Fifth, the number of included studies for several cancer types was extremely small, and this low number might weaken the reliability of the overall results. Finally, only the evidence for the correlation study was provided by this meta-analysis, which cannot be simply interpreted as a causal relationship.

\section{Conclusion}

Our meta-analysis revealed that a high level of AST/ALT ratio before treatment is significantly associated with worse OS, CSS, and RFS in patients with solid tumors. We conclude that serum AST/ALT ratio can serve as a reliable index for risk stratification of cancer patients in clinical settings because it has low cost and involves convenient operations. However, further high-quality clinical research must be performed to validate the prognostic role of pretreatment AST/ALT ratio in patients with malignancies due to the aforementioned limitations.

\section{Acknowledgments}

This study was supported by National Natural Science Foundation of China (Grant 81702270), Guangdong Province Science and Technology Development of Special Funding Support (Grant 2016A020215151), and Competitive Project of Financial Special Funds for Science and Technology of Zhanjiang City (Grant 2018A01026).

\section{Disclosure}

The authors report no conflicts of interest in this work.

\section{References}

1. Bray F, Ferlay J, Soerjomataram I, Siegel RL, Torre LA, Jemal A. Global cancer statistics 2018: GLOBOCAN estimates of incidence and mortality worldwide for 36 cancers in 185 countries. CA Cancer J Clin. 2018;68:394-424. doi:10.3322/caac.21492

2. Mäbert K, Cojoc M, Peitzsch C, Kurth I, Souchelnytskyi S, Dubrovska A. Cancer biomarker discovery: current status and future perspectives. Int $J$ Radiat Biol. 2014;90:659-677. doi:10.3109/ 09553002.2014.892229

3. Borrebaeck CA. Precision diagnostics: moving towards protein biomarker signatures of clinical utility in cancer. Nat Rev Cancer. 2017;17:199-204. doi:10.1038/nrc.2016.153

4. Füzéry AK, Levin J, Chan MM, Chan DW. Translation of proteomic biomarkers into FDA approved cancer diagnostics: issues and challenges. Clin Proteomics. 2013;10:13-27. doi:10.1186/1559-0275-10-13

5. Stocken DD, Hassan AB, Altman DG, et al. Modelling prognostic factors in advanced pancreatic cancer. $B r J$ Cancer. 2008;99:883-893. doi:10.1038/sj.bjc. 6604568

6. Thornburg JM, Nelson KK, Clem BF, et al. Targeting aspartate aminotransferase in breast cancer. Breast Cancer Res. 2008;10:R84. doi: $10.1186 /$ bcr 2154

7. De Ritis F, Coltorti M, Giusti G. An enzymic test forthe diagnosis of viral hepatitis; the transaminase serum activities. Clin Chim Acta. 1957;2:70-74.

8. Botros M, Sikaris KA, Lu ZX, McNeil A. The short-term prognostic usefulness of the De Ritis ratio. Clin Biochem Rev. 2013;34:S18.

9. Zoppini G, Cacciatori V, Negri C, et al. The aspartate aminotransferase-to-alanine aminotransferase ratio predicts all-cause and cardiovascular mortality in patients with type 2 diabetes. Medicine (Baltimore). 2016;95:e4821. doi:10.1097/ MD.0000000000004864

10. Rief P, Pichler M, Raggam R, et al. The AST/ALT (De-Ritis) ratio: a novel marker for critical limb ischemia in peripheral arterial occlusive disease patients. Medicine (Baltimore). 2016;95:e3843. doi:10.1097/ MD.0000000000004864

11. Gao F, Chen C, Lu J, et al. De Ritis ratio (AST/ALT) as an independent predictor of poor outcome in patients with acute ischemic stroke. Neuropsychiatr Dis Treat. 2017;13:1551-1557. doi:10.2147/NDT. S139316 
12. Kimm H, Kim S, Jee SH. The independent effects of cigarette smoking, alcohol consumption, and serum aspartate aminotransferaseon the alanine aminotransferase ratio in Korean men for the risk for esophageal cancer. Yonsei Med J. 2010;51:310-317. doi:10.3349/ ymj.2010.51.3.310

13. Sekar RR, Patil D, Baum Y, et al. A novel preoperative inflammatory marker prognostic score in patients with localized and metastatic renal cell carcinoma. Asian J Urol. 2017;4:230-238. doi:10.1016/j. ajur.2017.04.002

14. Steininger M, Winter MP, Reiberger T, et al. De-Ritis ratio improves long-term risk prediction after acute myocardial infarction. J Clin Med. 2018;7:E474. doi:10.3390/jcm7120474

15. Moher D, Shamseer L, Clarke M, et al. Preferred reporting items for systematic review and meta-analysis protocols (PRISMA-P) 2015 statement. Syst Rev. 2015;4:1. doi:10.1186/2046-4053-4-1

16. Peters JL, Sutton AJ, Jones DR, Abrams KR, Rushton L. Performance of the trim and fill method in the presence of publication bias and between-study heterogeneity. Stat Med. 2007;26:4544-4562. doi: $10.1002 / \operatorname{sim} .2889$

17. Ha YS, Kim SW, Chun SY, et al. Association between De Ritis ratio (aspartate aminotransferase/alanine aminotransferase) and oncological outcomes in bladder cancer patients after radical cystectomy. BMC Urol. 2019;19:10. doi:10.1186/s12894-019-0439-7

18. Kang M, Yu J, Sung HH, et al. Prognostic impact of the pretreatment aspartate transaminase/alanine transaminase ratio in patients treated with first-line systemic tyrosine kinase inhibitor therapy for metastatic renal cell carcinoma. Int J Urol. 2018;25:596-603. doi:10.1111/iju.13574

19. Canat L, Ataly HA, Agalarov S, Alkan I, Altunrende F. The effect of AST/ALT (De Ritis) ratio on survival and its relation to tumor histopathological variables in patients with localized renal cell carcinoma. Int Braz J Urol. 2018;44:288-295. doi:10.1590/S16775538.IBJU.2017.0173

20. Miyake H, Matsushita Y, Watanabe H, et al. Significance of De Ritis (aspartate transaminase/alanine transaminase) ratio as a significant prognostic but not predictive biomarker in Japanese patients with metastatic castration- resistant prostate cancer treated with cabazitaxel. Anticancer Res. 2018;38:4179-4185. doi:10.21873/anticanres.12711

21. Chen J, Huang C, Chen K, et al. Overexpression of LBH is associated with poor prognosis in human hepatocellular carcinoma. Onco Targets Ther. 2018;11:441-448. doi:10.2147/OTT.S152953

22. Gu L, Wang Z, Chen L, et al. A proposal of post-operative nomogram for overall survival in patients with renal cell carcinoma and venous tumor thrombus. J Surg Oncol. 2017;115:905-912. doi:10.1002/jso.24575

23. Ishihara H, Kondo T, Yoshida K, et al. Evaluation of preoperative aspartate transaminase/alanine transaminase ratio as an independent predictive biomarker in patients with metastatic renal cell carcinoma. Clin Genitourin Cancer. 2017;15:598-604. doi:10.1016/j.clgc.2017.04.011

24. Wang H, Fang K, Zhang J, et al. The significance of De Ritis (aspartate transaminase/alanine transaminase) ratio in predicting pathological outcomes and prognosis in localized prostate cancer patients. Int Urol Nephrol. 2017;49:1391-1398. doi:10.1007/s11255-017-1618-7

25. Gorgel SN, Kose O, Koc EM, Ates E, Akin Y, Yilmaz Y. The prognostic significance of preoperatively assessed AST/ALT (De Ritis) ratio on survival in patients underwent radical cystectomy. Int Urol Nephrol. 2017;49:1577-1583. doi:10.1007/s11255-017-1648-1

26. Liu C, Jia BS, Zou BW, et al. Neutrophil-to-lymphocyte and aspartate-to-alanine aminotransferase ratios predict hepatocellular carcinoma prognosis after transarterial embolization. Medicine (Baltimore). 2017;96:e8512. doi:10.1097/MD.0000000000008512

27. Cho YH, Hwang JE, Chung HS, et al. The De Ritis (aspartate transaminase/alanine transaminase) ratio as a predictor of oncological outcomes in patients after surgery for upper urinary tract urothelial. Int Urol Nephrol. 2017;49:1383-1390. doi:10.1007/ s11255-017-1613-Z
28. Nishikawa M, Miyake H, Fujisawa M. De Ritis (aspartate transaminase/alanine transaminase) ratio as a significant predictor of recurrence-free survival in patients with upper urinary tract urothelial carcinoma. Urol Oncol. 2016;34:417. doi:10.1016/j. urolonc.2016.04.001

29. Lee H, Choi YH, Sung HH, et al. De Ritis Ratio (AST/ALT) as a significant prognostic factor in patients with upper tract urothelial cancer treated with surgery. Clin Genitourin Cancer. 2017;15:e379e385. doi:10.1016/j.clgc.2016.08.023

30. Lee H, Lee SE, Byun SS, Kim HH, Kwak C, Hong SK. De Ritis ratio (aspartate transaminase/alanine transaminase ratio) as a significant prognostic factor after surgical treatment in patients with renal cell carcinoma. BJU Int. 2017;119:261-267. doi:10.1111/bju.13545

31. Takenaka Y, Takemoto N, Yasui T, et al. Transaminase activity predicts survival in patients with head and neck cancer. PLoS One. 2016;11:e0164057. doi:10.1371/journal.pone.0164057

32. Bezan A, Mrsic E, Krieger D, et al. The preoperative AST/ALT (De Ritis) ratio represents a poor prognostic factor in a cohort of patients with nonmetastatic renal cell carcinoma. J Urol. 2015;194:30-35. doi:10.1016/j.juro.2015.01.083

33. Xie X, Yao M, Chen X, et al. Reduced red blood cell count predicts poor survival after surgery in patients with primary liver cancer. Medicine(Baltimore). 2015;94:e577. doi:10.1097/ MD.0000000000000874

34. Kao WY, Chiou YY, Hung HH, et al. Risk factors for long-term prognosis in hepatocellular carcinoma after radiofrequency ablation therapy the clinical implication of aspartate aminotransferase-platelet ratio index. Eur $J$ Gastroenterol Hepatol. 2011;23:528-536. doi:10.1097/MEG.0b013e328346d529

35. Liu L, Wang W, Zhang Y, et al. Declined preoperative aspartate aminotransferase to neutrophil ratio index predicts poor prognosis in patients with intrahepatic cholangiocarcinoma after hepatectomy. Cancer Res Treat. 2018;50:538-550. doi:10.4143/ crt.2017.106

36. Ou Y, Huang J, Yang L. The prognostic significance of pretreatment serum $\gamma$-glutamyltranspeptidase in primary liver cancer: a metaanalysis and systematic review. Biosci Rep. 2018;38:pii: BSR20181058. doi:10.1042/BSR20181058

37. Metwalli AR, Rosner IL, Cullen J, et al. Elevated alkaline phosphatase velocity strongly predicts overall survival and the risk of bone metastases in castrate-resistant prostate cancer. Urol Oncol. 2014;32:761-768. doi:10.1016/j.urolonc.2014.03.024

38. Feldstein AE, Canbay A, Angulo P, et al. Hepatocyte apoptosis and fas expression are prominent features of human nonalcoholic steatohepatitis. Gastroenterology. 2003;125:437-443.

39. Wu J, Chen M, Liang C, Su W. Prognostic value of the pretreatment neutrophil-to-lymphocyte ratio in cervical cancer: a meta-analysis and systematic review. Oncotarget. 2017;8:13400-13412. doi:10.18632/ oncotarget.14541

40. Liu Y, Zhao P, Cheng M, et al. AST to ALT ratio and arterial stiffness in non-fatty liver Japanese population: a secondary analysis based on a cross-sectional study. Lipids Health Dis. 2018;17:275. doi:10.1186/ s12944-018-0920-4

41. Pilarczyk K, Carstens H, Heckmann J, et al. The aspartate transaminase/alanine transaminase (DeRitis) ratio predicts mid-term mortality and renal and respiratory dysfunction after left ventricular assist device implantation. Eur J Cardiothorac Surg. 2017;52:781-788. doi:10.1093/ejcts/ezx247

42. Dang CV. Links between metabolism and cancer. Genes Dev. 2012;26:877-890. doi:10.1101/gad.189365.112

43. Sookoian S, Pirola CJ. Liver enzymes, metabolomics andgenome-wide association studies: from systems biology to thepersonalized medicine. World J Gastroenterol. 2015;21:711-725. doi:10.3748/wjg.v21.i3.711 
44. Proctor MJ, Morrison DS, Talwar D, et al. An inflammationbasedprognostic score (mGPS) predicts cancer survival independent of tumour site: a Glasgow Inflammation Outcome Study. $\mathrm{Br}$ J Cancer. 2011;104:726-734. doi:10.1038/sj.bjc.6606087

45. Friday E, Oliver R 3rd, Welbourne T, Turturro F. Glutaminolysis and glycolysis regulation by troglitazone in breast cancer cells: relationship to mitochondrial membrane potential. $J$ Cell Physiol. 2011;226:511-519. doi:10.1002/jcp.22360

46. Conde VR, Oliveira PF, Nunes AR, et al. The progression froma lower to a higher invasive stage of bladder cancer is associated with severe alterations in glucose and pyruvate metabolism. Exp Cell Res. 2015;335:91-98. doi:10.1016/j. yexcr.2015.04.007
47. Sosa V, Moliné T, Somoza R, Paciucci R, Kondoh H, LLeonart ME. Oxidative stress and cancer: an overview. Ageing Res Rev. 2013;12:376-390. doi:10.1016/j.arr.2012.10.004

48. Taniguchi K, Karin M. NF- $\kappa \mathrm{B}$, inflammation, immunity and cancer: coming of age. Nat Rev Immunol. 2018;18:5-18. doi:10.1038/nri.2017.97

49. Ruart M, Chavarria L, Campreciós G, et al. Impaired endothelial autophagy promotes liver fibrosis by aggravating the oxidative stress response during acute liver injury. J Hepatol. 2019;70:458-469. doi:10.1016/j.jhep.2018.10.015

50. Zheng J, Seier K, Gonen M, et al. Utility of serum inflammatory markers for predicting microvascular invasion and survival for patients with hepatocellular carcinoma. Ann Surg Oncol. 2017;24:3706-3714. doi:10.1245/ s10434-017-6060-7
OncoTargets and Therapy

\section{Publish your work in this journal}

OncoTargets and Therapy is an international, peer-reviewed, open access journal focusing on the pathological basis of all cancers potential targets for therapy and treatment protocols employed to improve the management of cancer patients. The journal also focuses on the impact of management programs and new therapeutic

\section{Dovepress}

agents and protocols on patient perspectives such as quality of life, adherence and satisfaction. The manuscript management system is completely online and includes a very quick and fair peer-review system, which is all easy to use. Visit http://www.dovepress.com/ testimonials.php to read real quotes from published authors. 